\title{
Yod
}

Revue des études hébraïques et juives

$18 \mid 2013$

Le Proche-Orient ancien à la lumière des sciences sociales

\section{Que reste-t-il des théories sociologiques de George Mendenhall et de Norman Gottwald sur l'installation des tribus israélites au pays de} Canaan?

What valuability for the sociological theories of George Mendenhall and Norman Gottwald concerning the installation of Israelite Tribes in Canaan?

מה נותר מן התאוריות הסוציולוגיות של מנדנהל וגוטוולד על התיישבות

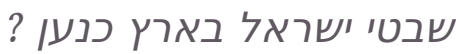

Daniel Bodi

\section{(2) OpenEdition}

Journals

Édition électronique

URL : https://journals.openedition.org/yod/1761

DOI : 10.4000/yod. 1761

ISSN : 2261-0200

Éditeur

INALCO

\section{Édition imprimée}

Date de publication : 1 septembre 2013

ISBN : 978-2-85837-207-8

ISSN : 0338-9316

\section{Référence électronique}

Daniel Bodi, «Que reste-t-il des théories sociologiques de George Mendenhall et de Norman Gottwald sur l'installation des tribus israélites au pays de Canaan ? ", Yod [En ligne], 18 | 2013, mis en ligne le 08 juillet 2013, consulté le 08 juillet 2021. URL : http://journals.openedition.org/yod/1761 ; DOI : https:// doi.org/10.4000/yod.1761

Ce document a été généré automatiquement le 8 juillet 2021.

\section{(c) (†) \&}

Yod est mis à disposition selon les termes de la Licence Creative Commons Attribution - Pas d'Utilisation Commerciale 4.0 International. 


\section{Que reste-t-il des théories sociologiques de George Mendenhall et de Norman Gottwald sur l'installation des tribus israélites au pays de Canaan?}

What valuability for the sociological theories of George Mendenhall and Norman Gottwald concerning the installation of Israelite Tribes in Canaan?

מה נותר מן התאוריות הסוציולוגיות של מנדנהל וגוטוולד על התיישבות

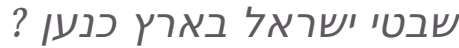

Daniel Bodi

Historiens, sociologues, archéologues et biblistes ont des opinions divergentes sur l'implantation des tribus israélites au pays de Canaan. Celles-ci reposent sur les différentes traditions hébraïques qui présentent côte à côte la conquête militaire et l'infiltration pacifique. Jusqu'à la fin $\mathrm{du} \mathrm{xx}^{\mathrm{e}}$ siècle, il y avait trois théories principales sur l'installation des tribus israélites au pays de Canaan : celle de William F. Albright et de l'« école de Baltimore ", la théorie formulée par les historiens allemands Albrecht Alt et Martin Noth, modifiée par Manfred Weippert, ainsi que la théorie sociologique avancée par George Mendenhall et Norman Gottwald. Actuellement, ce sont les études de l'archéologue Israël Finkelstein et de l'historien Mario Liverani qui prédominent. Si elles incorporent des éléments des théories précédentes, elles en critiquent toutefois les limites.

\section{William Albright et l'« école de Baltimore »}

2 L'« école de Baltimore ", constituée autour des travaux de William F. Albright, George Ernest Wright, John Bright et Paul Lapp ${ }^{1}$, a avancé une explication qu'on pourrait 
désigner comme l'« hypothèse de l'invasion ». Elle postule la prise du pouvoir par les tribus hébraïques venant de la Transjordanie à travers la conquête militaire des villes cananéennes et des territoires en Cisjordanie. Albright tenta de faire concorder les récits bibliques avec les découvertes archéologiques des strates successives comportant des traces de destruction. Il essaya d'interpréter les fouilles archéologiques dans ce sens afin d'étayer son hypothèse de la conquête du pays de Canaan au XIII siècle av. n. è., telle qu'elle est décrite dans les récits bibliques des livres de Josué et des Juges (Jos 8.1-35 [Aï] ; 10.1-14 [Gibeôn]; 11.1-14 [Merom]; Jg 1.8 [les tribus de Juda et de Siméon contre les Cananéens]; Jg 5, le cantique de Déborah qui décrit la victoire des tribus de Naphtali et Zébulon dans la partie sud de la plaine de Jezréel²). Suivant cette approche, les grands centres urbains cananéens, tels que Lakhish, Debir, Eglon (Tell elHesi) et Bethel, furent détruits par les tribus israélites ${ }^{3}$. Durant les années 1950, un autre élément fut ajouté grâce aux excavations du site de Hașôr par Yigal Yadin ${ }^{4}$, montrant que la ville fut détruite à la fin du xIII siècle av. n. è. Toutefois, ces découvertes archéologiques ne sont pas sans équivoque et certaines des identifications des sites établies par Albright se sont révélées fausses. Comme le remarque Moshe Weinfeld, "même s'il était démontré qu'une ville particulière eut été détruite au XIII ${ }^{e}$ siècle av. n. è., on ne saurait être sûr que la destruction eut été l'acte des Israélites. Cette destruction aurait pu être l'œuvre des Égyptiens, ou des Peuples de la mer, ou bien encore des Cananéens luttant les uns contre les autres $»^{5}$.

De plus, le site de Debir n'est plus identifié à Tell Beit Mirsim, comme Albright le suggérait, mais plutôt à Khirbet Rabud ${ }^{6}$. Cet ajustement affaiblit son explication archéologique parce qu'à la différence de Beit Mirsim, qui présente des signes de destruction datant du XIII ${ }^{e}$ siècle, Khirbet Rabud ne montre aucun signe de destruction datant de cette époque. En outre, il n'y a pas de preuve que la ville de Lakhish (Tell edDuweir) fut détruite au XIII ${ }^{\mathrm{e}}$ siècle. Par contre, il est évident que le site en question était sous domination égyptienne au début du XII ${ }^{\mathrm{e}}$ siècle (l'époque de Ramsès III) ${ }^{7}$. À propos des villes de Jéricho et d'Aï, il n'y a pas de signes indiquant la destruction de leurs murailles à l'âge du bronze récent. Les récits de la conquête de ces villes sont étiologiques, ce qui invalide leur historicité. En effet, la destruction de nombreuses villes datant de l'âge du bronze récent ne peut être attribuée à un seul événement ou à un envahisseur unique.

Malgré les difficultés dans l'interprétation de données archéologiques, on devrait accepter le fait qu'un grand nombre de villes cananéennes furent détruites au XIII ${ }^{\mathrm{e}}$ et au $\mathrm{XII}^{\mathrm{e}}$ siècles av. n. è., ce qui confirme le déroulement de certaines opérations militaires au moment où les Israélites prirent possession du territoire. Qui détruisit ces villes demeure, néanmoins, une question discutable.

\section{L'« infiltration pacifique »}

5 L'hypothèse de l'« infiltration pacifique » connaît deux versions. La plus ancienne, proposée par Albrecht Alt ${ }^{8}$ et Martin Noth ${ }^{9}$ et adoptée avec quelques modifications par Manfred Weippert ${ }^{10}$, pose comme postulat l'arrivée pacifique des tribus israélites nomades en provenance de territoires semi-désertiques et infiltrant le pays de Canaan à la recherche de nouveaux pâturages. Elles devinrent progressivement un peuple sédentaire. La nouvelle version, proposée par Israël Finkelstein, apporte une modification en suggérant que des groupes tribaux pastoraux composés d'anciens 
Cananéens occupèrent de manière graduelle et complémentaire les hautes terres. Ces tribus évoluèrent progressivement, devenant des Israélites.

6 Dans cette optique, la " conquête » se déroula sans faire usage de la force. Les Israélites s'installèrent dans les territoires qui n'étaient pas incorporés dans le système des citésÉtats ou bien dans des territoires peu peuplés. Ceci semble confirmé par le traité conclu, selon Jos 9, avec les Gabaonites (=el Djib, $10 \mathrm{~km}$ au nord, nord-ouest de Jérusalem) et par l'absence de combats en Palestine centrale.

7 M. Weippert conclut son analyse des diverses théories concernant l'installation des tribus israélites en affirmant que l'hypothèse d'Alt peut être considérée comme la plus objective et celle d'Albright comme la plus subjective du fait qu'elle impose aux textes bibliques ainsi qu'aux sources archéologiques des interprétations harmonisatrices. Albright et ses disciples sont souvent obligés d'adapter les traditions bibliques aux données archéologiques et les résultats des fouilles archéologiques aux affirmations des textes. Weippert souligne qu'il n'y a pas de faits archéologiques tels quels, mais seulement des faits interprétés. Puisqu'aucun document n'a été découvert parmi les excavations, l'archéologie ne peut rien dire sur les causes de la destruction de ces villes, ni sur les auteurs de leur destruction ${ }^{11}$. Weippert apporte quelques modifications à l'hypothèse d'Alt-Noth. En se référant aux lettres d'El-Amarna et à la liste royale égyptienne des noms des localités, il affirme que les régions montagneuses du pays de Canaan étaient faiblement peuplées. Elles pouvaient ainsi offrir assez de place aux éleveurs de petit bétail ${ }^{12}$. La première implantation pacifique fut conduite par les tribus issues de Léa et celles qui y sont associées, à savoir le groupe de Bilhah et de Zilpah. Ce n'est que dans un deuxième temps que s'installèrent les tribus issues de Rachel, pratiquant le culte de Yahvé et adhérant à l'idée d'une guerre portée en son nom. Commence ainsi la période d'occupation armée à laquelle renvoie un certain nombre de textes bibliques. Ce faisant, Weippert incorpore dans son explication des éléments de l'hypothèse de Rudolf Smend sur les tribus guerrières de Rachel comme porteuses de la tradition des guerres de Yahvé, le dieu des armées ${ }^{13}$.

Cette opinion semble partiellement confirmée par des textes comme Jos 17.14-18 où on préconise le déboisement comme moyen d'agrandir la région d'installation, car dans la vallée, les Cananéens étaient trop forts et possédaient des chars en fer les rendant inattaquables (voir aussi Jg 1.19). Les données archéologiques confirment aussi l'argument de base de cette école: de nombreux vestiges d'habitation dense correspondant à la période d'installation ont été découverts dans les collines d'Éphraïm, de Manassé et de Juda ${ }^{14}$. Les fouilles ont montré que durant le XII ${ }^{\mathrm{e}}$ siècle av. $\mathrm{n}$ è., un grand nombre de villages apparurent soudain dans les régions des collines où des villages cananéens prospéraient déjà. De même, elles ont révélé un système de fortifications situé au sommet des montagnes, sur une bande territoriale allant de la côte phénicienne jusqu'aux hautes montagnes de la Galilée orientale. Ceci renforce l'hypothèse de R. de Vaux selon laquelle la destruction de Hașôr a été causée par l'expansion des tribus israélites en Galilée. Cependant, selon V. Fritz l'hypothèse de l'infiltration pacifique n'explique pas la chute des cités-États cananéennes, car l'effondrement de la civilisation cananéenne au bronze récent ne présente pas les conditions requises pour le processus d'installation ${ }^{15}$. 


\section{L'hypothèse du « bouleversement social »}

9 La troisième explication ou l'hypothèse du «bouleversement social» («social upheaval ») suit une approche sociologique et anthropologique. Elle est avancée par George Mendenhall, Norman Gottwald et Cornelis de Geus ${ }^{16}$ et c'est elle qui nous intéresse tout particulièrement ici. Pour présenter l'explication sociohistorique de N. Gottwald au sujet de l'installation des tribus israélites au pays de Canaan, il faut d'abord présenter les opinions de son précurseur, G. Mendenhall, dont il s'est inspiré. Cet exercice historiographique est nécessaire. On s'aperçoit aujourd'hui que l'hypothèse Gottwald-Mendenhall trouve confirmation lorsque nous la confrontons aux nouvelles données épigraphiques, historiques et archéologiques du Proche-Orient ancien. Ainsi, dans une perspective historiographique, les critiques de Hauser et de Thompson ${ }^{17}$ dévoilent de plus grandes faiblesses que les hypothèses qu'ils essaient d'invalider. En effet, les théories de Mendenhall et de Gottwald ont été reprises et popularisées par les historiens récents qui les ont aussi reformulées. Il faut reconnaître néanmoins que les deux théories, celle de Mendenhall et celle de Gottwald, souffraient du style tout à fait particulier de leurs auteurs. Mendenhall, un luthérien, avait tendance à adopter un style moralisateur provoquant la critique exacerbée de Jack Sasson $^{18}$, tandis que Gottwald, un activiste politique engagé, cite allégrement Karl Marx, Mao Tse Dong ainsi que des sociologues ou économistes dont il trouva les théories pertinentes pour son argument. En utilisant parfois un jargon marxiste, Gottwald cite des auteurs habituellement absents du domaine des études bibliques. Mais, si on fait abstraction de leur style particulier, on découvre que l'essentiel de leurs théories est toujours pertinent et résiste, d'après moi, à l'épreuve du temps.

En 1962, Mendenhall formula une hypothèse sociohistorique expliquant qu'Israël devait son origine à la révolte des paysans et des bergers de Palestine contre les dirigeants tyranniques des villes. Il la proposa comme alternative à la théorie de A. Alt et M. Noth, alors dominante dans l'étude de l'ancien Israël, selon laquelle celui-ci se constitua par l'infiltration pacifique des tribus nomades. Mendenhall affirmait plutôt que la prise du pays de Canaan par les Israélites impliquait un conflit et considérait que les guerriers d'Israël étaient à l'origine des paysans cananéens indigènes qui avaient rejoint le groupe de l'exode passé par le Sinaï et avaient adopté leur culte de Yahvé. Ces paysans s'étaient volontairement séparés de la société cananéenne et ne devaient pas être perçus comme des nomades envahisseurs :

Cela devient plus clair si l'on suppose que les premiers Israélites avaient été tout d'abord sous la domination des villes cananéennes et avaient réussi par la suite à s'en soustraire. Une fois devenus indépendants, ils avaient résolu de ne pas reconstruire le même type de société au pouvoir central, avide de prestige, auquel ils venaient d'échapper, bien que cela représentât une tentation constante (p. ex. Abimélek) à laquelle ils succomberaient finalement (sous Salomon) ${ }^{19}$.

11 Mendenhall appuie son modèle des révoltes locales des Cananéens sur certaines données des lettres d'El-Amarna. Dans la lettre EA 138, 71-73 par exemple, le roitelet Rib-Addi de Byblos se plaint que seule une moitié de sa ville lui est soumise ainsi qu'au gouvernement égyptien suzerain, tandis que l'autre moitié a pris le parti du chef amorrite en révolte. Inspirés par les exemples des communautés religieuses d'Israël, les paysans cananéens locaux détrônèrent leurs rois et adoptèrent un style de société plus égalitaire en accord avec l'idéologie israélite. 
12 Mendenhall affirme que la conquête de la Palestine par les tribus israélites n'était pas l'affaire d'un seul groupe ethnique, Israël, détruisant un autre groupe, les Cananéens, mais qu'il s'agissait plutôt des masses indigènes des Cananéens se révoltant contre l'aristocratie régnante composée pour la plupart de conquérants ayant envahi le pays auparavant : les Hittites, les Hivvites, les Girgashites, les Perizzites et les Jébuséens ${ }^{20}$. Ce point de vue n'a pas été confirmé par la prospection archéologique menée par Israël Finkelstein et ses étudiants de l'Université de Tel Aviv, lesquels trouvèrent très peu de différences entre les poteries dont se servaient les populations qui vivaient dans les hautes terres et celles vivant dans la plaine ${ }^{21}$. Ceci constitue un indice qu'en fin de compte, un même peuple vivait dans les deux régions. Subsiste cependant une différence majeure relevée par M. Liverani et qui représente un important indicateur de distinction ethnique : aucun ossement de porc n'a été trouvé dans les villages des hautes terres, laissant penser que ces habitants avaient adopté peut-être un régime alimentaire différent de celui des Cananéens de la vallée ${ }^{22}$.

13 Mendenhall formula un argument supplémentaire, décrié lui aussi, car trop inhabituel. Il nota que, dans la stratigraphie des fouilles en Palestine, les couches de destruction surgissent avec une fréquence presque régulière, tous les 250-300 ans, ce qui équivaut à 10 générations humaines (dix fois trente ans), d'où le titre inhabituel de son livre $L a$ dixième génération ${ }^{23}$. Il élabora sa théorie en prenant en compte la périodisation archéologique présumée du Levant entre l'âge du bronze et l'âge du fer. Chaque région présente une périodisation spécifique et au Levant elle suit ce schéma : l'âge du bronze ancien ([2100-1760 av. n. è.] commençant avec la migration amorrite et finissant avec l'incendie de Mari par Hammourabi de Babylone); l'âge du bronze moyen I-II, ([1760-1500] qui prend fin avec l'expulsion des Hyksos d'Égypte) et l'âge du bronze récent ([1460-1170] qui s'achève avec l'incendie d'Ugarit lors de l'invasion par les Peuples de la mer et la fin de la domination égyptienne au pays de Canaan. Plusieurs villes cananéennes furent incendiées soit par les Philistins soit par certaines tribus israélites); l'âge du fer I-III (1100-605). D'habitude, les limites entre ces différentes périodes sont marquées par les couches de cendre qui s'insèrent dans la succession des strates archéologiques. Mendenhall affirmait avoir mis en évidence une "périodisation tenant compte de la documentation archéologique $»^{24}$, ce que la communauté scientifique avait rejeté comme étant une généralisation péremptoire. D’après lui, ces destructions sont le résultat d'implosions internes des sociétés locales provoquées par la désintégration des relations humaines, des institutions sociales et des principes moraux, aboutissant à un changement rapide de pouvoir. Qui plus est, l'intervalle de 300 ans correspondrait, au nord d'Israël, à l'époque comprise entre le règne de Saül (cca 1020-1010) et la chute de Samarie (722). Dans l'histoire de Jérusalem et du royaume de Juda, le même intervalle de 300 ans peut être observé entre le début du règne de Roboam, fils de Salomon, en 930, et l'arrivée de Nabuchodonosor II en 605 av. n. è. Les prophètes hébreux auraient donc joué le rôle d'observateurs décrivant et mettant en garde contre ce processus qui devait conduire à l'implosion des sociétés israélites.

Comment la révolte des paysans fut-elle déclenchée? Mendenhall attribue un rôle majeur à l'alliance faite au mont Sinaï entre Yahvé et un petit groupe, une "multitude mélangée », fuyant la servitude en Égypte. Cette notion d'alliance dotée d'une idéologie égalitaire attira les paysans cananéens locaux en créant des liens de solidarité et de loyauté avec les nouveaux venus. Cette alliance était séduisante pour tous les groupes qui souffraient sous le joug de l'asservissement à un pouvoir tyrannique. Certains 
termes de cette alliance étaient attirants, par exemple l'idée que chaque fermier recevrait sa terre comme un fief de Yahvé au lieu de l'obtenir d'un chef de guerre despotique, ou bien l'idée qu'une norme sociale s'applique à tous les membres de la société et non à l'avantage des riches et au détriment des pauvres ${ }^{25}$. Selon Mendenhall, toutes les organisations sociales se caractérisent par la formation de liens affectifs sur une base idéologique. "Parmi tous les traits idéologiques qui semblent être universels figurent les religions, la moralité, la langue et la loi. ${ }^{26}$ L'alliance aurait fourni le cadre à la constitution du liant religieux entre les anciens Israélites. Ceux-ci représentaient un catalyseur social au pays de Canaan. Il semble que l'apparition de la petite communauté religieuse d'Israël polarisa la population dans tout le pays; certains se joignirent à eux, les autres, principalement les rois et leurs sympathisants, les attaquèrent. Puisque les rois furent défaits et chassés, ces événements fournirent le support de la tradition selon laquelle les Cananéens et les Amorrites furent tous chassés ou exterminés en masse et selon laquelle seuls les Israélites étaient restés, constituant ainsi la majorité de la population partout dans le pays ${ }^{27}$.

Comme l'a indiqué A. J. Hauser ${ }^{28}$, les données archéologiques peuvent montrer que la destruction d'une ville a eu lieu ou que le site a été habité durant une certaine période, mais elles ne peuvent indiquer les causes spécifiques de la destruction. Toutefois, Mendenhall décide d'interpréter les indices de destruction comme des signes de décadence interne due à l'implosion cyclique de la société provoquée par la fracture sociale et par l'insensibilité des riches et des puissants envers les pauvres et les opprimés.

16 Contrairement à Mendenhall, Gottwald distingue entre une tradition du Sinaï et une tradition de l'exode et de l'installation au pays de Canaan. En effet, il adopte à ce propos les conclusions de Gerhard von Rad dans ses travaux sur l'Hexateuque où il montre que c'est seulement à l'époque postexilique que ces traditions ont été associées, comme en témoigne par exemple le texte de $\mathrm{Ne} 9^{29}$.

De plus, le parallèle établi par Mendenhall entre les 'apiru et les Hébreux, affirmant qu'ils étaient " pratiquement des synonymes " $^{30}$, est aujourd'hui abandonné. Comme l'a bien observé Weippert, Mendenhall accorde « une trop grande importance à la volonté d'appartenir à la catégorie des 'apiru. Il me semble évident que l'entrée dans cette catégorie de déclassés devrait être considérée, selon les textes qui semblent l'indiquer entre les lignes, comme un malheur [...] C'est la pression externe qui en est la cause et non pas le libre choix $»^{31}$.

Selon l'ouvrage majeur de Gottwald, The Tribes of Yahweh, les anciens Israélites représentaient une assemblée dépareillée de Cananéens marginaux, comprenant les paysans «féodalisés » (Hupšu), les mercenaires 'apiru, les aventuriers, les bergers en transhumance, les fermiers organisés en tribus, les nomades éleveurs de bétail (š3św = šosu) et probablement aussi les artisans itinérants et les prêtres désaffectés. Israël émanait d'une scission radicale d'avec la société cananéenne et non pas d'une invasion, ni d'une migration externe.

Ainsi, le dogme du herem associé aux guerres de Yahvé avait une raison d'être clairement socio-économique. La classe dominante cananéenne menacée espérait discréditer les révolutionnaires israélites comme anarchistes. Par conséquent, les Israélites devaient s'abstenir de destructions délibérées et de fêtes gloutonnes après les pillages. Le programme socio-économique était le suivant : premièrement, repousser la zone de contrôle des cités-États cananéennes pour se débarrasser définitivement des 
impôts opprimants, de la pratique de la corvée ainsi que du service militaire. Deuxièmement, établir un ordre social autonome et viable fondé sur l'égalité d'accès aux sources de base de telle façon que nul ne vive des surplus produits par les autres. Si on tient compte de ces objectifs et des méthodes de la révolution israélite, tout le butin et les personnes capturées pendant une bataille devaient être sagement repartis afin d'affermir la conception sociale des insurgés. Les rois et leurs affiliés, les fonctionnaires, les chefs militaires, les propriétaires, les marchands, les artisans de produits de luxe, tous devaient être tués, expulsés ou convertis afin d'accepter un rôle plus modeste et non élitiste dans la société israélite. Tous les autres Cananéens avaient une place légitime au sein d'Israël, ceux qui appartenaient aux couches sociales inférieures.

On apprend du récit biblique que Josué et David coupèrent les jarrets des chevaux capturés. Cette mutilation était nécessaire parce que les chevaux appartenaient à la machinerie de guerre ennemie et ne pouvaient être réutilisés par les Israélites. Par contre, les bœufs faisaient partie du butin, car ils nourrissaient les paysans en cultivant et en fertilisant leurs terres, en portant des fardeaux et en contribuant de temps en temps à leur nourriture.

Gottwald offre une interprétation originale de la "guerre sainte", ou plus correctement de la guerre de Yahvé. Selon lui, la guerre sainte représentait un mouvement révolutionnaire des couches inférieures de la société du pays de Canaan contre les agents d'exploitation tels que les rois cananéens et l'élite sociale qui formait la classe dominante ${ }^{32}$. Les guerres de Yahvé sont en fait bien préparées à l'avance, grâce au " programme socio-économique révolutionnaire israélite». Malheureusement pour la théorie de Gottwald, il n'y a aucune donnée extrabiblique ni même archéologique qui corroborerait une révolution si bien conçue.

Que signifie le fait que les métaux étaient destinés au trésor de Yahvé ou bien encore qu'ils étaient herem, "consacrés » à Yahvé ? Cela pourrait vouloir dire qu'ils étaient placés dans une réserve commune à multiples usages. Cette réserve pouvait contenir des objets de culte, elle pouvait servir à financer les prêtres ou à fournir les outils et les armes nécessaires. De même pouvait-elle remplir une fonction sociale comme servir à l'achat d'aliments de base en cas de famine, au paiement des rançons pour recouvrer ou garder les terres, à la sauvegarde des gens menacés de confiscation ou d'esclavage ${ }^{33}$.

Gottwald fonde son interprétation générale sur une exégèse minutieuse et philologique des textes hébreux. Dans le cantique de Déborah en Jg 5.6-7, la TOB propose la traduction traditionnelle de ce texte :

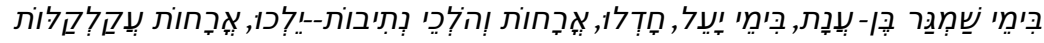

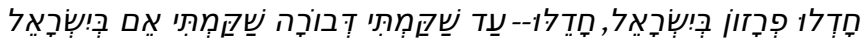

Aux jours de Shamgar, fils de 'Anat, aux jours de Yaël, avaient cessé (hādelû) les caravanes et ceux qui voyageaient allaient par des chemins détournés. Des villages étaient abandonnés (ḥâdelû), ils étaient abandonnés (hāāellû) en Israël, jusqu'à ce que tu te sois levée, Déborah, jusqu'à ce que tu te sois levée, mère en Israël.

Dans un autre passage, $1 \mathrm{~S} 2.5$, où figure le terme hādal, Gottwald indique correctement l'existence d'un topos littéraire du « renversement radical de fortune » :

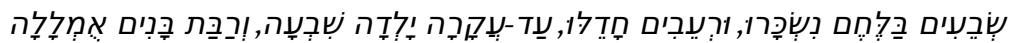
Les rassasiés s'embauchent pour du pain, mais les affamés cessent (hāāellû) de travailler. La femme stérile enfante sept fois, mais la mère de nombreux enfants se flétrit (Bible de Jérusalem). 
Gottwald, la clé pour déchiffrer ces textes opaques et pour clarifier la situation historique, incomprise par les traducteurs, se trouve dans l'étude philologique de D. Winton Thomas et de M. L. Chaney ${ }^{34}$. Ces spécialistes de l'hébreu ont tenté de montrer qu'il y avait en fait deux racines hāadal. Le hădal familier et plus fréquent qui signifie " cesser " et une deuxième racine hădal qui signifie "devenir gros, repu ou potelé ", expression qui correspond au terme arabe de même origine qui signifie «devenir potelé ou charnu ». Cette distinction entre les deux racines se retrouve dans le texte massorétique qui signale que la deuxième racine est un verbe statif et place un dagesh inhabituel dans la troisième consonne dans l'une des deux occurrences en Jg 5.7 (hâadêllû) et dans l'occurrence unique en $1 \mathrm{~S}$ 2.5. Si le terme hădēllû en $1 \mathrm{~S} 2.5$ est interprété comme "avoir cessé", le verset n'a pas de sens ou bien demande un complément pour assurer le sens : "Les rassasiés s'embauchent pour du pain, mais les affamés cessent... » (mais « cessent » de faire quoi, par rapport à quoi ?). En revanche, si hāàelllû en $1 \mathrm{~S} 2.5$ signifie "est devenu gros » et le 'ad suivant est traduit comme "butin», le parallélisme poétique concorde avec les autres descriptions du "renversement radical de fortune" indiquées dans l'ensemble poétique en $1 \mathrm{~S} 2.5$ : "Ceux qui étaient rassasiés s'embauchent pour du pain, tandis que ceux qui avaient faim ont grossi du butin. La stérile a enfanté sept fois, mais la mère de nombreux enfants se flétrit. » La New American Bible semble avoir incorporé cette lecture et traduit de la façon suivante : «tandis que les affamés se gavent du butin » ("while the hungry batten on spoil »).

En acceptant le fait que le vocable hādal a deux sens, Jg 5.6-7 peut être compris comme l'expression, artistiquement travaillée, d'un jeu de mots s'appuyant sur les deux racines hādal et sur la juxtaposition ironique de leurs deux significations.

Les caravanes ont cessé (hāalal I), les voyageurs (« les rois » en LXX ${ }^{\mathrm{A}}$ ) prenaient des sentiers détournés. Les villageois d'Israël se gavaient (hādal II) du butin, lorsque tu t'es levée, Déborah, lorsque tu t'es levée, mère en Israël !

Gottwald offre une explication sociohistorique de ces lignes difficilement compréhensibles. L'ironie exprimée dans la juxtaposition de hădal I, « cesser », et de hādal II, "grossir », met en valeur des sujets radicalement opposés : d'une part les entreprises impériales cananéennes ${ }^{35}$ et, d'autre part, l'armée israélite égalitaire formée de paysans. Les premiers (les campagnes militaires ou caravanes) ont "cessé " tandis que les deuxièmes (l'armée d'Israël) "grossissent en se gavant du butin» provenant des caravanes pillées et des entrepôts militaires pris ${ }^{36}$.

Gottwald conclut donc que le cantique de Déborah

projette deux mondes radicalement différents du point de vue de l'organisation sociale, le monde hiérarchique opposé au monde égalitaire, « la cité » contre « la campagne ", deux mondes qui se confrontent par l'intermédiaire de deux structures absolument différentes : une armée professionnelle suréquipée en armements perfectionnés face à une armée citoyenne, modestement et irrégulièrement armée, mais rusée et munie d'un élan extraordinaire et dotée de flexibilité tactique. ${ }^{37}$

La conclusion de Gottwald est peut-être correcte, néanmoins elle interpelle par le vocabulaire utilisé, ressemblant plutôt à un paragraphe tiré d'un livre sur la révolution américaine décrivant les tactiques des fermiers américains contre les tuniques rouges, soldats du roi d'Angleterre. Cependant, cela peut être attribué à son désir de présenter ses théories d'une façon pédagogiquement adaptée à ses étudiants nord-américains ${ }^{38}$. 
30 À propos du terme per rāzôn, « les paysans » ou « les villageois », présent dans le cantique de Déborah, Liverani explique qu'il s'agit de villages ouverts, perāzôt, qui ne sont pas protégés par des murailles, ce que confirment les études archéologiques de Finkelstein. Ces villages sont érigés en opposition aux cités cananéennes protégées par des remparts et des triples portes. Liverani y reconnaît lui aussi un conflit socioéconomique entre les deux groupes sociaux ${ }^{39}$.

31 La manière dont les idées de Mendenhall et de Gottwald furent reprises dans le débat actuel sur l'histoire ancienne d'Israël peut être illustrée par l'explication de Liverani au sujet de l'installation des tribus israélites dans les hautes terres de la Cisjordanie. Liverani décrit la situation dans cette région au moment où il n'y avait que deux citésÉtats exerçant le pouvoir palatial, l'une à Sichem et l'autre à Jérusalem. Liverani remarque qu'à l'époque d'El-Amarna déjà, au XIV siècle av. n. è., Sichem et Jérusalem étaient différentes des autres cités-États de la plaine. Elles avaient des tendances expansionnistes au détriment des petits villages aux alentours. À l'appui de ce point de vue, il cite $E A$ 280, 30-35 où le dirigeant de la ville de Gat dit :

Lab'ayu (roi de Sichem) est mort, lui qui prenait nos villes, mais maintenant c'est

'Abdi-Heba (roi de Jérusalem) qui est comme un second Lab'ayu et qui prend nos villes. Déborah. Dans mon article sur la critique proférée par le prophète Ézéchiel à l'encontre de la monarchie judéenne au moment de la chute de Jérusalem ${ }^{41}$, j'ai analysé le topos littéraire proche-oriental du « renversement radical de fortune » relevé par Gottwald. On peut lire en Éz 7.27 :

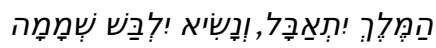

Le roi sera dans le deuil, le chef/prince se revêtira de désolation.

Selon cette affirmation, le roi et le nassis désarroi par le deuil et le vêtement de misère et de désolation. Voir les aristocrates et les riches habillés en guenilles est un topos connu dans la littérature égyptienne ; il sert à décrire un bouleversement social et la détérioration radicale des conditions de vie. Le papyrus très fragmentaire de Leyde intitulé «Les admonestations d'Ipu-wer $»^{42}$, datant de la XIX dynastie (1350-1220 av. n. è.), décrit les bouleversements survenus lors de la Première Période Intermédiaire ayant suivi la chute de l'Ancien Empire. Les envahisseurs asiatiques occupèrent le delta du Nil et renversèrent l'ordre en vigueur. Le sage Ipu-wer remarque : "Ceux qui possédaient des robes sont (maintenant) en haillons. » C'est probablement dans le même sens que nous devons comprendre 
l'inscription de Deir 'Alla rédigée en paléo-araméen et datant de 760 av. n. è. Dans le premier fragment, ligne 12, nous lisons : lnš'.'zr.qrn " pour un chef / prince un pagne de toile ». En Job 12.18 le maître suprême de l'univers provoque le renversement de fortune pour les riches : «Il desserre la ceinture des rois et noue un pagne ('ēzôr) à leurs reins." Tous ces textes décrivent le renversement radical de fortune, résultat de bouleversements sociaux.

Le renvoi aux lettres d'El-Amarna relatant les conflits entre les palais des villes cananéennes et les paysans des villages représente un des aspects durables de l'analyse de Mendenhall, aspect repris récemment par l'assyriologue Liverani dans son approche de l'histoire ancienne d'Israël.

Bien que Finkelstein critique l'hypothèse de Mendenhall et de Gottwald ${ }^{43}$, il adopte néanmoins deux de leurs prémisses: premièrement, l'existence d'une crise sociale servant de catalyseur aux changements sociaux et, deuxièmement, l'idée que les Israélites émergèrent de l'intérieur de la société cananéenne. Le point faible de Finkelstein réside dans le fait que toute son explication se base uniquement sur l'archéologie et sur sa façon d'interpréter les données archéologiques. Il n'incorpore pas les données des textes d'El-Amarna ${ }^{44}$ et a tendance à rejeter le témoignage des textes bibliques. Cependant, les historiens contemporains tels que Mario Liverani, au lieu de rejeter les trois théories - celle de la conquête, celle de l'infiltration pacifique et celle du bouleversement social - trouvent un élément de vérité dans chacune d'elles et incorporent dans leurs explications ce que chacune a de pertinent.

\section{NOTES}

1. W. F. Albright, "The Israelite Conquest of Canaan in the Light of Archaeology", BASOR 74, 1939, pp. 11-23; The Biblical Period from Abraham to Ezra, New York, Harper, 1963, pp. 23-34; G. Ernest Wright, Biblical Archaeology, Philadelphie, Westminster, 1962, pp. 69-85; J. Bright, A History of Israël, Philadelphie, Westminster, 1959, pp. 117-127; P. W. Lapp, "The Conquest of Palestine in the Light of Archaeology", Concordia Theological Monthly 38, 1967, pp. 283-300.

2. Le cantique de Déborah en Jg 5 n'énumère que dix tribus: Éphraïm, Benjamin, Machir, Zébulon, Issachar, Ruben, Gilead, Dan, Asher et Naphtali. Juda et Siméon ne sont pas mentionnés.

3. A. Malamat, "Israelite Conduct of War in the Conquest of Canaan", dans F. M. Cross (éd.), Symposia Celebrating the Seventy-Fifth Anniversary of the Founding of the American Schools of Oriental Research (1900-1975), Cambridge, MA, Harvard University Press, 1979, pp. 35-56; B. S. J. Isserlin, “The Israelites' Conquest of Canaan: A Comparative Review of the Arguments Applicable", Palestine Exploration Quarterly 115, 1983, pp. 85-94.

4. Y. Yadin, Hazor with a Chapter on Israelite Megiddo, Londres, Oxford University Press, 1972.

5. M. Weinfeld, The Promise of the Land: The Inheritance of the Land of Canaan by the Israelites, Berkeley, University of California Press, 1993, p. 102, no. 4.

6. M. Kochavi, “'Khirbet Rabûd'= Debir", Tel Aviv: Journal of the Institute of Archaeology of Tel Aviv University 1, 1974, pp. 2-33.

7. O. Tufnell, Lachish, The Bronze Age 4, Londres, Oxford University Press, 1958, p. 37 et p. 98 . R. Giveon, “An Inscription of Rameses III from Lachish”, Tel-Aviv 10, 1983, pp. 176-177; D. Ussishkin, 
“Excavations at Tel Lachish, 1978-1983: Second Preliminary Report”, Tel-Aviv 10, 1983, pp. 169-170.

8. A. Alt, »Die Landnahme der Israeliten In Palästina« in Kleine Schriften zur Geschichte des Volkes Israel, Munich, C. B. Beck, 1953, vol. 1, p. 85-125; „Erwäungen über die Landnahme der Israeliten in Palätina«, in Kleine Schriften, vol.1, p.126-175; "Josua« in Kleine Schriften, vol. 1, p. 176-192; "The Settlement of the Israelites in Palestine" in Essays on Old Testament History and Religion, Garden City, N.Y., Doubleday, 1968, pp. 75-221.

9. M. Noth, Das Buch Josua, HAT I/7, Göttingen, Vandenhoeck \& Rupprecht, 1953. Geschichte Israels, Göttingen, Vandenhoeck \& Rupprecht, 1954, p. 67-82 = The History of Israel, New York, Harper, 1960, pp. 68-84.

10. Selon Alt et Noth, l'installation des tribus israélites a eu lieu sans combat dans les régions peu habitées des collines de Galilée, Éphraïm et Juda. Pour une discussion détaillée de cette hypothèse voir $\mathrm{M}$. Weippert, Die Landnahme der israelitischen Stämme in der neuren wissenschaftlichen Diskussion, FRLANT 92, Göttingen, Vandenhoeck \& Ruprecht, 1967, p. 14-51 = The Settlement of the Israelite Tribes in Palestine : A Critical Survey of Recent Debate, SBT 2/21, London, SCM Press, 1971.

11. M. Weippert, The Settlement of the Israelite Tribes in Palestine, op. cit. pp. 124-132.

12. Ibid., p. 140.

13. R. Smend, Jahwekrieg und Stämmebund. Erwägungen zur ältesten Geschichte Israels, FRLANT 84, Göttingen, Vandenhoeck \& Ruprecht, 1963 = Yahweh War and Tribal Confederation. Reflections upon Israel's Earliest History, tr. angl. M. G. Rogers, Nashville, Abingdon, 1970, p. 101. Smend proposa l'hypothèse selon laquelle les tribus de Rachel (Joseph [= Manassé et Éphraïm] et Benjamin) étaient porteuses de la tradition des guerres de Yahvé. Cependant, les tribus de Léa constituées par une coalition de six tribus, Ruben, Siméon, Lévi, Juda, Zébulon et Issachar, étaient déjà pacifiquement installées au pays de Canaan avant la période de la « conquête ».

14. M. Kochavi, "The Israelite Settlement in Canaan in the Light of Archaeological Survey", in J. Aviram et al. (éds), Proceedings of the International Congress on Biblical Archaeology, 1-10 April 1984, Jérusalem, Israel Exploration Society, 1985, pp.54-60 et I. Finkelstein, The Archaeology of the Israelite Settlement, Jérusalem, Israel Exploration Society, 1988.

15. V. Fritz, "Conquest or Settlement? The Early Iron Age in Palestine", Biblical Archaeologist 50, 1987, pp. 84-100, spéc. p. 84.

16. G. E. Mendenhall, “The Hebrew Conquest of Palestine', BA 25, 1962, pp. 66-87; N. K. Gottwald, The Tribes of Yahweh, New York, Orbis Books, 1979, pp. 543-550: "Sociological Settings of 'Holy War' and Herem"; C. H. L. de Geus, The Tribes of Israel, Assen-Amsterdam, Van Gorcum, 1976.

17. A. J. Hauser, “Israel's Conquest of Palestine: A Peasants' Rebellion?”, JSOT 7, 1978, pp. 2-19; T. L. Thompson, “Historical Notes on 'Israel's Conquest of Palestine: A Peasant's Rebellion?", JSOT 7, 1978, pp. 20-27.

18. J. Sasson, "Review of G. Mendenhall, The Tenth Generation", JBL 93, 1974, pp. 294-296.

19. G. E. Mendenhall, "The Hebrew Conquest of Palestine", Biblical Archaeologist 25, 1962, pp. 66-87, ici p. 77.

20. G. E. Mendenhall, The Tenth Generation: The Origins of the Biblical Tradition, Baltimore, The Johns Hopkins University Press, 1973, p. 225.

21. I. Finkelstein, The Archaeology of the Israelite Settlement, tr.angl. par D. Saltz, Jérusalem, Israel Exploration Society, 1988; I. Finkelstein (éd.), Archaeological Survey of the Hill Country of Benjamin, Jérusalem, Israel Antiquities Authority, 1993.

22. M. Liverani, La Bible et l'invention de l'histoire, Paris, Bayard, 2008, p. 89.

23. G. E. Mendenhall, The Tenth Generation, op. cit. pp. 216-217.

24. G. E. Mendenhall, "Between Theology and Archaeology", JSOT 7, 1978, pp. 28-34, spéc. p. 30.

25. G. E. Mendenhall, "The Hebrew Conquest of Palestine", pp. 76-79.

26. G. E. Mendenhall, "Between Theology and Archaeology", p. 32. 
27. G. E. Mendenhall, "The Hebrew Conquest of Palestine", p. 81, et p. 84: "Local populations which became Israelite by 'conversion' continued to value their own traditions, poetry, historical narratives, and customs. These are only incidentally 'ortsgebundene Traditionen' (traditions bound to geographical locations); they are traditions which were preserved because there is a continuity of population, and a continuity also of every feature of culture which was not a flagrant violation of the religious obligation to Yahweh."

28. A. J. Hauser, "Israel's Conquest of Palestine: A Paesants' Rebellion?", p. 6.

29. G. von Rad, "The Form-Critical Problem of the Hexateuch" in The Problem of the Hexateuch and Other Essays, New York, McGraw-Hill, 1966, pp. 1-78; voir aussi N. K. Gottwald, The Tribes of Yahweh, op. cit. pp. 79-82: "Sequential Articulation of the Traditions".

30. G. E. Mendenhall, "The Hebrew Conquest of Palestine", p. 71 et The Tenth Generation, op. cit. p. 140.

31. M. Weippert, The Settlement of the Israelite Tribes in Palestine, op. cit. p. 66.

32. N. K. Gottwald, The Tribes of Yahweh, op. cit. p. 547.

33. Ibid., p. 549.

34. D. Winton Thomas, "Some Observations on the Hebrew Root hdl", VTS, 4, 1958, p. 8-16 et "CHDL-II in Hebrew", Catholic Biblical Quarterly (CBQ) 24, 1962, p. 154; Ph. Calderone, "CHDL-II in Poetic Texts" CBQ 23, 1961, pp. 451-460 ; M. L. Chaney, Textual, Philological, and Sociological Studies in Judges 5, with Special Reference to the Verbal Occurrences of HDL in Biblical Hebrew, Unpubl. Ph.D. diss., Harvard University, 1976.

35. Ces entreprises sont perçues soit comme des «caravanes" soit comme des «campagnes militaires ", le terme «expéditions " qui traduit l'hébreu 'orāhôt pouvant signifier les deux, comme le mot akkadien correspondant Harranu.

36. N. K. Gottwald, The Tribes of Yahweh, op. cit. pp. 504-506.

37. Ibid., p. 507.

38. Cf. le titre de la conférence de Gottwald faite en 2006 au congrès annuel de la Society of Biblical Literature à Washington D.C., "Structure and Origin of the Early Israelite and Iroquois Confederacy", dans le cadre de la section travaillant sur "Hebrew Bible and Political Theory".

39. Liverani, La Bible et l'invention de l'histoire, op. cit. p. 130.

40. W. L. Moran, Les lettres d'El-Amarna, tr. fr. D. Collon et H. Cazelles, LAPO 13, Paris, Cerf, 1987, p. 502 et p. 471.

41. D. Bodi, «Le prophète critique la monarchie : le terme nāssī' chez Ézéchiel », dans A. Lemaire (éd.), Prophètes et rois. Bible et Proche-Orient, Paris, Cerf, 2001, p. 249-257, spéc. p. 253-254.

42. J. A. Wilson, "The Admonitions of Ipu-wer", ANET, p. 442.

43. I. Finkelstein et N. A. Silberman, La Bible dévoilée, Paris, Bayard, 2002, p. 127.

44. Voir la critique de cette faiblesse dans l'argument de Finkelstein faite par son collègue de Tel Aviv, N. Na'aman, “The Contribution of the Amarna Letters to the Debate on Jerusalem's Political Position in the Tenth Century BCE", BASOR 304, 1996, pp. 17-27.

\section{RÉSUMÉS}

Historiens, sociologues, archéologues et biblistes ont des opinions divergentes sur l'implantation des tribus israélites au pays de Canaan. Jusqu'à la fin $\mathrm{du} \mathrm{xx}^{\mathrm{e}}$ siècle, il y avait trois théories principales sur l'installation des tribus israélites au pays de Canaan : celle de William F. Albright 
et de l'école de Baltimore, celle formulée par les historiens allemands Albrecht Alt et Martin Noth ainsi que la théorie sociologique avancée par George Mendenhall et Norman Gottwald. Le présent article examine la valeur scientifique actuelle de cette dernière.

Historians, sociologists, archaeologists and Bible scholars diverge consistently in their opinion concerning the Israelite installation in Canaan. Three theories where prevailing up to the end of the 20th century: the theory of William F. Albright and the Baltimore School, the theory of the German historians Albrecht Alt and Martin Noth, as well as the sociological theory of George Mendenhall and Norman Gottwald. The present article aims to analyze the contemporary scientific value of this last one.

\section{INDEX}

Mots-clés : tribus israélites, installation en Canaan, ethnogenèse, Mendenhall George E. (1916-), Gottwald Norman (1926-)

Keywords : Israelite Tribes, installation in Canaan, ethno-genesis, Mendenhall George E. (1916-), Gottwald Norman (1926-), Mediterranean, Middle East

Index géographique : Méditerranée, Proche-Orient

\section{מילות מפתח}

שבטי ישראל, המזרח התיכון, הים התיכון, ההתישבות בארץ כנען, לידתו של עם:

\section{AUTEUR}

DANIEL BODI

Université Paris 8 